$12-1992$

\title{
On the Difficulty of Manhattan Channel Routing
}

Ronald I. Greenberg

Loyola University Chicago, Rgreen@luc.edu

Joseph JaJa

Sridhar Krishnamurthy

Follow this and additional works at: https://ecommons.luc.edu/cs_facpubs

Part of the Theory and Algorithms Commons, and the VLSI and Circuits, Embedded and Hardware Systems Commons

Author Manuscript

This is a pre-publication author manuscript of the final, published article.

\section{Recommended Citation}

Information Processing Letters, Volume 44, Issue 5, 21 December 1992, Pages 281-284.

This Article is brought to you for free and open access by the Faculty Publications and Other Works by Department at Loyola eCommons. It has been accepted for inclusion in Computer Science: Faculty Publications and Other Works by an authorized administrator of Loyola eCommons. For more information, please contact ecommons@luc.edu.

\section{(c) $($ () $\ominus$}

This work is licensed under a Creative Commons Attribution-Noncommercial-No Derivative Works 3.0 License. (c) Elsevier, 1992. 


\title{
On the Difficulty of Manhattan Channel Routing
}

\author{
Ronald Greenberg ${ }^{1,2,4}$ \\ Joseph JáJáa, $\hat{a}^{1,3,5}$ \\ Sridhar Krishnamurthy $y^{1,3}$
}

\begin{abstract}
We show that channel routing in the Manhattan model remains difficult even when all nets are single-sided. Given a set of $n$ single-sided nets, we consider the problem of determining the minimum number of tracks required to obtain a dogleg-free routing. In addition to showing that the decision version of the problem is $N P$-complete, we show that there are problems requiring at least $d+\Omega(\sqrt{n})$ tracks, where $d$ is the density. This existential lower bound does not follow from any of the known lower bounds in the literature.
\end{abstract}

Key Words: VLSI channel routing, combinatorial problems, computational complexity, lower bounds, and $N P$-completeness.

\section{Introduction}

We are given two horizontal lines whose separation may be adjusted to form the boundaries of a rectilinear grid, and a set of $n$ nets. Each net consists of two terminals located at grid points on the boundaries. The region between the top boundary and the bottom boundary is referred to as the channel. The channel routing problem requires that we specify paths along grid-line segments within the channel that connect the two terminals of each net. The goal is to minimize the total number of horizontal grid lines (tracks) in the channel (or in the decision version of the problem to test whether $k$ tracks suffice). We consider the Manhattan model, where the horizontal segments of a wire are on one layer and the vertical segments are on the other layer. Thus, wire segments may cross, since they will be on different layers, but different wires may not otherwise share any gridpoint. When a connection is desired at a grid point between a horizontal and a vertical segment of the same wire, it is achieved by an explicit contact cut.

The first complexity result for the Manhattan channel routing problem was by LaPaugh [2], who showed that the problem is NP-complete when no doglegs are allowed. (The routing of a net has a dogleg if it uses more than one horizontal wire segment.) Szymanski showed that the problem remains $N P$-complete even when doglegs are allowed [3]. There are also two important lower bounds on the number of tracks required. Define the local density $d_{x}$ at horizontal coordinate $x$, to be the number of nets whose intervals contain $x$. The density $d$ is given by the maximum of

\footnotetext{
${ }^{1}$ Department of Electrical Engineering, University of Maryland, College Park, MD 20742.

${ }^{2}$ University of Maryland Institute for Advanced Computer Studies (UMIACS), College Park, MD 20742.

${ }^{3}$ Systems Research Center, University of Maryland, College Park, MD 20742.

${ }^{4}$ Partially supported by NSF grant CCR-9109550.

${ }^{5}$ Partially supported by NSF grants CCR-9103135 and CD-8803012.
} 
local densities over all columns in the channel. Clearly the number of horizontal tracks required for the layout must be at least $d$. Baker, Bhatt and Leighton [1] showed that by considering horizontal cuts along channel boundaries, one can obtain a second lower bound $f$ referred to as flux. The flux for a problem with $n$ nets can be as large as $\Theta(\sqrt{n})$.

In this paper, we consider the single-sided channel routing problem. This problem is the same as the problem discussed above except that nets are restricted to have both their terminals on the same horizontal boundary of the channel. We refer to nets with both terminals on the top (bottom) boundary as single-sided top (bottom) nets.

In what follows, we restrict attention to dogleg-free routing for the single-sided channel routing problem. In the next section, we show that the problem is $N P$-complete even though it appears simpler than the problem with general nets. In Section 3 we show that there are problems requiring $d+\Omega(\sqrt{n})$ tracks. This existential lower bound does not follow from the universal lower bound of $\max \{d, f\}$, since a problem with only single-sided nets that has flux $\Omega(\sqrt{n})$ must have density $\Omega(n)$.

\section{$2 \quad N P$-completeness of Single-Sided Channel Routing}

We show that the decision version of the single-sided channel routing problem is $N P$-complete by using LaPaugh's NP-completeness result for general 2-terminal nets.

Theorem 1 It is NP-complete to determine if single-sided channel routing problems are routable in a given number of tracks.

Proof: Given an instance of the general channel routing problem with $k$ tracks and a set of 2terminal nets, we produce an instance of the single-sided channel routing problem as follows. Let $n_{i}$ be a two-sided net with top terminal in column $l_{i}$ and bottom terminal in column $r_{i}$, with $l_{i}<r_{i}$. For each such $n_{i}$, let $d_{i}$ denote the local density between columns $l_{i}$ and $l_{i}+1$. Then add $2 k$ new columns immediately to the right of $l_{i}$, and replace $n_{i}$ by the following set of single-sided nets:

- a top net $t_{i}$ having terminals in columns $l_{i}$ and $l_{i}+k$

- a bottom net $b_{i}$ with terminals in columns $l_{i}+k+1$ and $r_{i}$

- $k-d_{i}$ top nets covering the following intervals: $\left\{\left[l_{i}+k-j, l_{i}+k+j+1\right], 1 \leq j \leq k-d_{i}\right\}$

For each net $n_{i}$ with $l_{i}>r_{i}$, we do the same transformation immediately to the left of $l_{i}$. Note that we need not consider $l_{i}=r_{i}$ since these trivial columns can be thrown away when considering dogleg-free routings. In Figure 1, we show how to replace a two-sided net with a set of single-sided nets, when $k=5$ and $d_{i}=2$.

It remains to show that the original channel routing problem can be routed using $k$-tracks, if and only if $k$-tracks suffice for the corresponding single-sided problem. For the 'only if' direction, observe that for any two-sided net $n_{i}$, the $\leq 2 k$ columns used to transform it to single-sided nets do not overlap with the columns used for any other transformation. Thus, there is room to place the additional $k-d_{i}$ nets, since the absence of doglegs ensures that the other nets use only a single track. For the 'if' direction, observe that for any $k$-track solution to the single-sided problem, the set of $k-d_{i}$ single-sided nets added for the transformation of two-sided net $n_{i}$ forces top net $t_{i}$ and bottom net $b_{i}$ to share the same track. Thus the routing for the two-sided net $n_{i}$ for the original problem can be obtained by combining the routings for $t_{i}$ and $b_{i}$. 


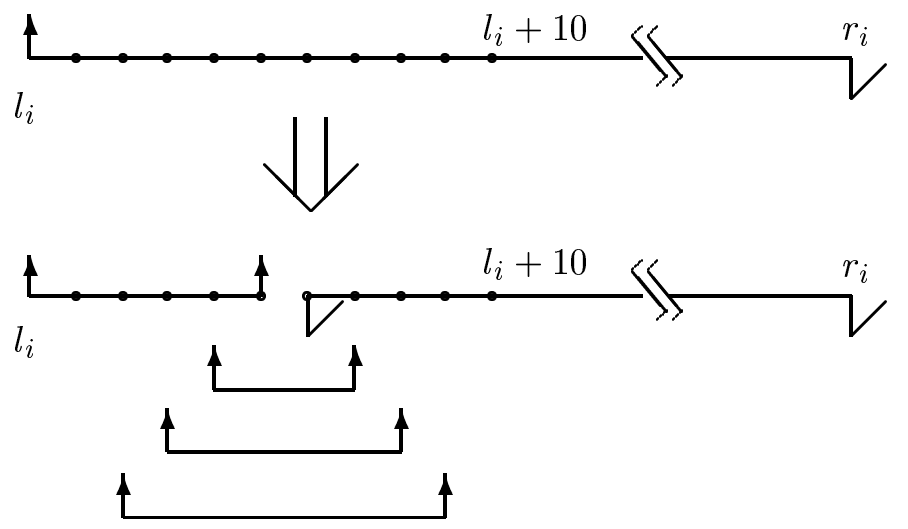

Figure 1: Replacing a two-sided net by a set of single-sided nets, for $k=5, d_{i}=2$

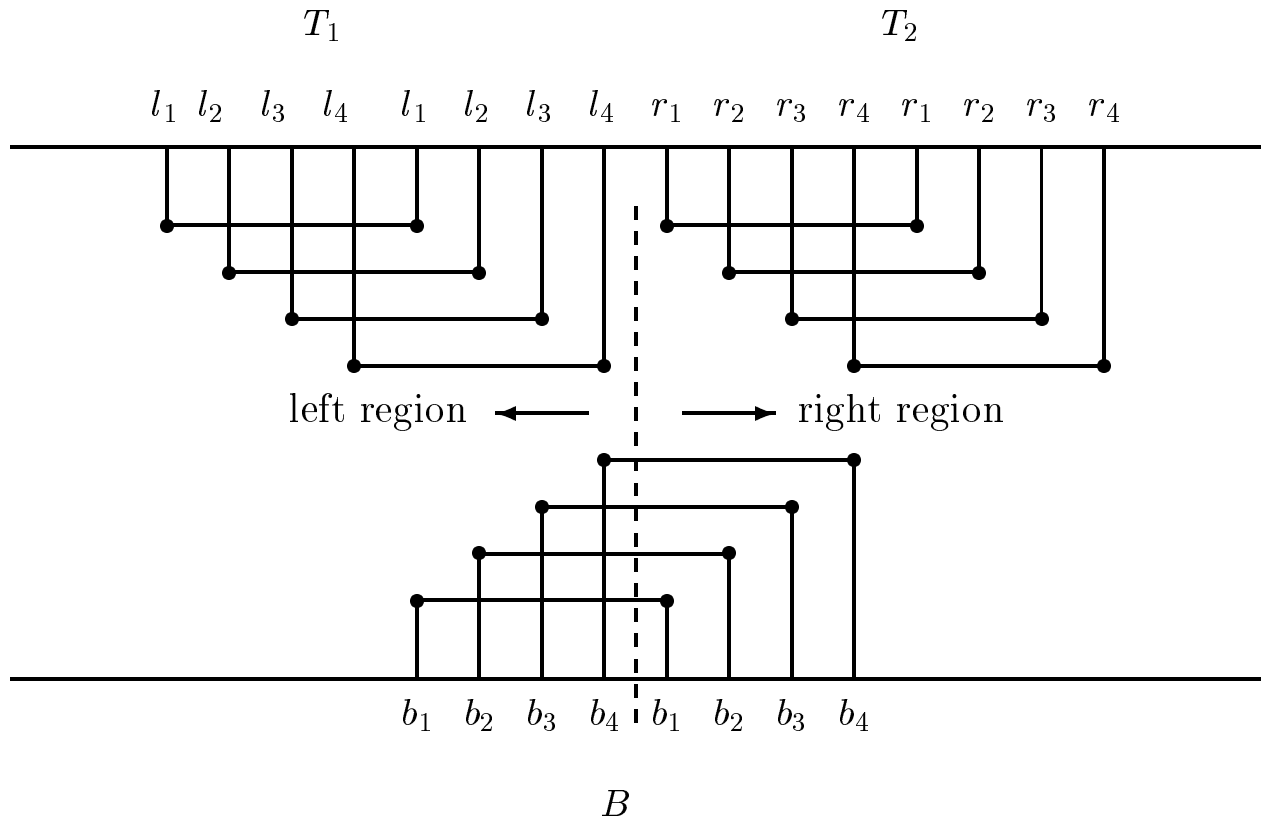

Figure 2: difficult example for $n=12$

\section{An Existential Lower Bound}

In this section, we show that when the layout is restricted to be dogleg-free there exist "difficult" instances of the single-sided channel routing problem, which require at least $d+\Omega(\sqrt{n})$ tracks.

A difficult example of $n$ nets consists of two sets of top nets, $T_{1}$ and $T_{2}$, and a set of bottom nets $B$. Each set contains $n / 3$ nets. Let $T_{1}=\left\{l_{1}, l_{2}, \ldots, l_{n / 3}\right\}, T_{2}=\left\{r_{1}, r_{2}, \ldots, r_{n / 3}\right\}$, and $B=$ $\left\{b_{1}, b_{2}, \ldots, b_{n / 3}\right\}$. The problem has columns numbered 1 through $4 n / 3$. For $1 \leq i \leq n / 3$, a top net $l_{i}$ has terminals at columns $i$ and $i+\frac{n}{3}$, a top net $r_{i}$ has terminals at columns $\frac{2 n}{3}+i$ and $n+i$, and a bottom net $b_{i}$ has terminals at columns $\frac{n}{3}+i$ and $\frac{2 n}{3}+i$. The density, $d$ for this problem equals $n / 3+1$. Figure 2 shows a difficult example for $n=12$ nets.

Theorem 2 Any routing solution for the difficult example with $n$ nets will use at least $n / 3+\sqrt{n / 3}$ tracks. 


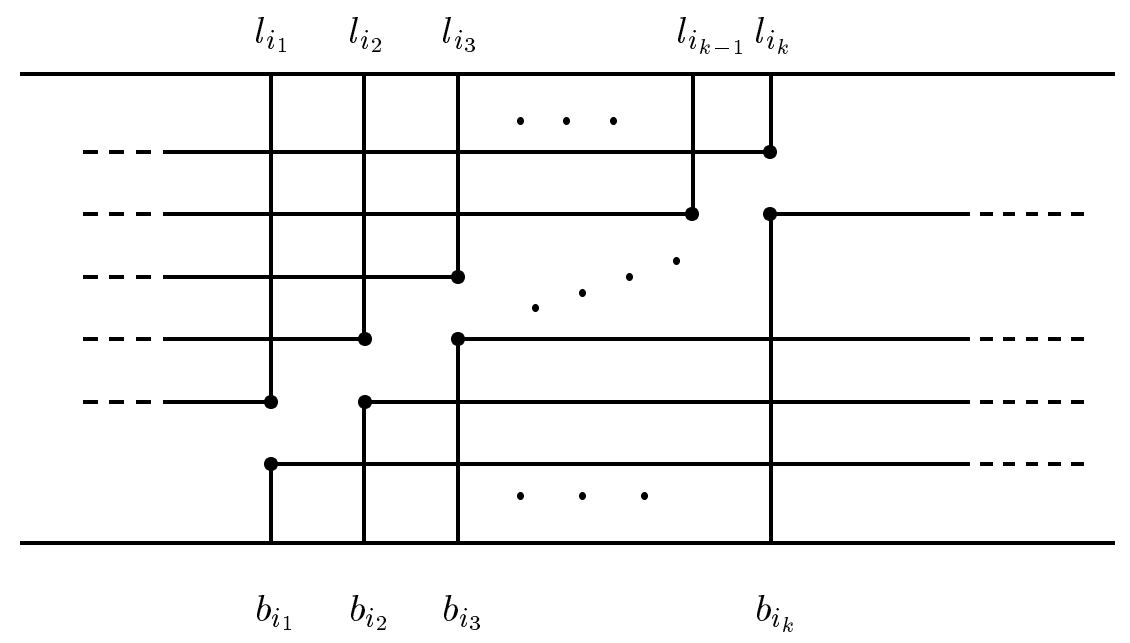

Figure 3: A chain from left region

Proof: The solution can be partitioned into two regions, the left region and the right region. The left region contains both terminals of each net in $T_{1}$ and the left terminals of all nets in $B$; the right region is defined similarly. (See Figure 2.) We begin by examining the routing in the left region. Partition the nets in $B$ and $T_{1}$ into a set of chains $\left\{L_{1}, L_{2}, \ldots, L_{p}\right\}$ by iterating over the following steps for $i=1,2, \ldots$, until each of the nets has been allocated to some $L_{i}$ :

Let $b_{i_{1}}$ denote the leftmost unallocated bottom net. Obtain an alternating series of bottom and top nets, $L_{i}=\left\{b_{i_{1}}, l_{i_{1}}, b_{i_{2}}, l_{i_{2}}, \ldots, b_{i_{k}}, l_{i_{k}}\right\}$, where $l_{i_{j}}$ is the top net with its right terminal in the same column as the left terminal of bottom net $b_{i_{j}}$, and $l_{i_{j}}$ shares a track with bottom net $b_{i_{j+1}}$ in the routing. The last net in the chain is a top net, $l_{i_{k}}$, and does not share a track with any bottom net in the routing. (See Figure 3.)

Observation 1 The number of tracks used by the nets in any chain $L_{i}, 1 \leq i \leq p$, equals $\left|L_{i}\right| / 2+1$. The total number of tracks used in the left region equals $\sum_{i=1}^{p}\left(\left|L_{i}\right| / 2+1\right)=n / 3+p$.

Similarly, we can partition the nets in $T_{2}$ and $B$ into a set of chains $R_{1}, R_{2}, \ldots R_{q}$, for the routing in the right region. Each chain $R_{i}=\left\{b_{i_{1}}, r_{i_{1}}, b_{i_{2}}, r_{i_{2}}, \ldots, r_{i_{m}}\right\}$, where $r_{i_{j}}$ is the top net (in $T_{2}$ ) with its left terminal in the same column as the right terminal of bottom net $b_{i_{j}}$, and $r_{i_{j}}$ shares a track with bottom net $b_{i_{j+1}}$. The last net in the chain is a top net, $r_{i_{m}}$, which does not share a track with any net from $B$. The total number of tracks used in the right region equals $\sum_{i=1}^{q}\left(\left|R_{i}\right| / 2+1\right)$ $=n / 3+q$.

Let $b_{i_{\alpha}}$ and $b_{i_{\beta}}$ be any two bottom nets belonging to $L_{i}$, with $b_{i_{\alpha}}$ appearing before $b_{i_{\beta}}$ in $L_{i}$. This implies that net $b_{i_{\beta}}$ is further to the right than $b_{i_{\alpha}}$ and is assigned a higher track in the routing solution. A similar but conflicting property holds for nets belonging to the same right chain, which leads us to the following observation.

Observation 2 Consider the set of bottom nets in any chain $L_{i}$, obtained from the left region. No two of these nets belong to the same chain $R_{j}$ in the right region. 
Let $\left|L_{m}\right|=\max \left\{\left|L_{i}\right|, 1 \leq i \leq p\right\}$. Clearly, $\left|L_{m}\right| \geq 2 n / 3 p$. From Observation 2 , this implies that $q$, the number of chains in the right region, is $\geq\left|L_{m}\right| / 2 \geq n / 3 p$. From Observation 1, the total number of tracks used in the routing solution is $\max \{n / 3+p, n / 3+q\} \geq \max \{n / 3+p, n / 3+n / 3 p\}$. This quantity is minimized, when $p=n / 3 p$, or $p=\sqrt{n / 3}$. Thus, the total number of tracks is $\geq n / 3+\sqrt{n / 3}=d+\Omega(\sqrt{n})$.

(A routing solution with $n / 3+\sqrt{n / 3}$ tracks can easily be obtained by selectively partitioning the nets in $T_{1}$ and $B$ into $\sqrt{n / 3}$ left chains, and the nets in $T_{2}$ and $B$ into $\sqrt{n / 3}$ right chains.)

\section{Conclusions}

We considered the channel routing problem under the dogleg-free Manhattan model with only single-sided nets. We showed that the problem is $N P$-complete and that there are problem instances which require at least $d+\Omega(\sqrt{n})$ tracks. An interesting open question, is whether the above results hold even when doglegs are allowed. The obvious strategy for the $N P$-completeness result would be to attempt an extension of Szymanski's reduction from 3SAT. But this reduction is complex, and it is not clear if it can be extended to single-sided channel routing. Another open question is whether the restriction to single-sided nets allows some simplification of the approximation algorithm of [1], which guarantees a layout using at most $d+O(f)$ tracks for 2 -terminal nets. For example, can we obtain a dogleg-free layout in $d+O(f)$ tracks?

\section{References}

[1] B. S. Baker, S. N. Bhatt, and T. Leighton. An approximation algorithm for manhattan routing. In F. P. Preparata, editor, VLSI Theory. Volume 2 of Advances in Computing Research, pages 205-229. JAI Press, 1984.

[2] A. S. LaPaugh. Algorithms for Integrated Circuit Layout: An Analytic Approach. PhD thesis, Department of Electrical Engineering \& Computer Science, Massachusetts Institute of Technology, Nov. 1980. MIT/LCS/TR-248.

[3] T. G. Szymanski. Dogleg channel routing is NP-complete. IEEE Trans. Computer-Aided Design of Integrated Circuits, CAD-4(1):31-41, Jan. 1985. 